Original Research

\title{
Diagnostic Approaches for the Assessment of Equine Chronic Pulmonary Disorders
}

\author{
Orsolya Kutasi DVM ${ }^{\mathrm{a}}$, Nandor Balogh PhD, dipl. ECVCP ${ }^{\mathrm{b}}$, Zoltan Lajos DVM ${ }^{\mathrm{c}}$, \\ Krisztina Nagy PhD ${ }^{\text {a }}$, Otto Szenci DVM, PhD, DSc, Dip. ECBHM ${ }^{\mathrm{a}}$ \\ a Clinic for Large Animals, Szent Istvan University, Üllö, Dora Major, Hungary \\ ${ }^{\mathrm{b}}$ PraxisLab Kft, Budapest, Hungary \\ ${ }^{\mathrm{C}}$ Duo-Bakt Veterinary Microbiology Laboratory, Budapest, Hungary
}

Keywords:

Chronic pulmonary disorders

Conditional inference tree

Diagnostics

\section{A B S T R A C T}

Even though the respiratory system is one of the most accessible organs for diagnostic testing, it is not always easy to define chronic lower airway disease in the horse. Diagnostic procedures performed by first opinion veterinarians in the field are often restricted to taking the history and performing clinical examination. Respiratory tract endoscopy, tracheal or bronchoalveolar lavage, and blood sampling are sometimes used but other specific ancillary examinations are seldom performed in stable settings. Therefore, our objectives were to evaluate the diagnostic value of different techniques and examination types routinely used in the diagnostic workup of chronic equine lower airway cases in both stable and clinical circumstances. Another aim of this study was to estimate the prevalence of different chronic pulmonary disorders among horses admitted to a Hungarian referral clinic. According to the conditional inference tree method, age of the horse, history, clinical examination, respiratory tract endoscopy, and bronchoalveolar lavage cytology proved to be the most valuable tools to define pathology. It was also concluded that in $22 \%$ of cases, more specific ancillary diagnostic modalities, unavailable for the field veterinarian, were needed to establish the final diagnosis. According to our study, the most frequently diagnosed chronic pulmonary disorders in Hungary are of noninfectious origin, principally recurrent airway obstruction. Regardless of the cause, and interestingly including recurrent airway obstruction as well, these diseases occur primarily during the warm months.

(c) 2011 Elsevier Inc. All rights reserved.

\section{Introduction}

After establishing a definite diagnosis in as many pulmonary cases as possible, a significant number of horses are always left with no definitive diagnosis even when using current understanding and available ancillary diagnostic techniques [1]. Although an accurate history and especially bronchoscopy can confirm the presence of pulmonary disease, pulmonary cytology forms a mainstay for

Corresponding author at: Orsolya Kutasi, DVM, Clinic for Large Animals, Faculty of Veterinary Science, Szent Istvan University, 2225, Üllő, Dora Major, Hungary.

E-mail address: kutasi.orsolya@aotk.szie.hu (O. Kutasi). diagnosing the specific chronic pulmonary disease using the criteria described in previously published data [2].

Chronic lower airway disorders can be of several origins such as allergy, hypersensitivity, infections, toxicity, loss of pulmonary vascular integrity, or neoplasia. One of the most commonly diagnosed chronic lower airway diseases is recurrent airway obstruction (RAO) [2], which is believed to be caused by an allergic reaction to inhaled molds and shares similarities with the noneosinophilic form of asthma in human beings [3-5]. Airway obstruction, inflammation, mucus accumulation, and tissue remodeling have been shown to contribute to the pathophysiology of RAO [6]. Airway obstruction causing typical labored breathing is reversible by controlling dust in the environment or using 
bronchodilators [6]. A mild form of lower airway inflammatory disease commonly encountered in young athletic horses has been recognized as a separate entity from RAO and is termed inflammatory airway disease (IAD) [7-9]. In the basis of clinical grounds; however, some have argued that, over time, horses with IAD may progress into RAO $[10,11]$. In the pathogenesis of IAD, a variety of causal agents might be involved, such as respirable organic and inorganic particles in stable dust [12], immunological factors, and infectious agents $[9,13]$. Although IAD is a nonseptic inflammation of the lower airways without any evidence of systemic signs of infection, in a previous study, a clear association was demonstrated between some infectious agents and the prevalence of IAD [13]. Infections causing lower airway disease in adult horses include viral, bacterial, fungal, and parasitic agents, and they more typically occur after a predisposing effect that suppresses pulmonary immunity like long-distance transport or strenuous exercise, resulting in systemic signs [14]. Exercise-induced pulmonary hemorrhage (EIPH) occurs in the majority of racehorses and is observed sporadically in many other sport horses that require strenuous exercise for short periods $[14,15]$. Proposed pathophysiological mechanisms include high pulmonary vascular pressures during maximal exercise as well as pulmonary inflammation or obstruction of the upper or lower airways [14,16,17]. Other lower airway disorders like granulomatous, neoplastic diseases, or interstitial pneumonias are rarely diagnosed in horses [14]. Differentiation between the aforementioned lower airway respiratory disorders on the basis of their flexible and ambiguous definitions can sometimes be difficult or even impossible. Clinical signs and the causal factors may overlap, or one of these disorders may induce the other. Because treatment and prognosis can significantly differ, an appropriate diagnosis is always necessary.

Our objectives were to evaluate the diagnostic value of different techniques and examination types used routinely in the diagnostic workup of chronic equine lower airway cases by field veterinarians and in clinical circumstances. Another aim of this study was to estimate the prevalence of different equine lower airway diseases among horses admitted to a Hungarian referral clinic.

\section{Materials and Methods}

The study was performed between July 2005 and August 2008, at the Clinic for Large Animals, Faculty of Veterinary Science, Szent István University. In total, 100 horses (25 stallions, 39 geldings, and 36 mares) of different the majority of cases, RAO and IAD may be differentiated on

breeds-61 Hungarian Half-breeds, 10 other European Half-breeds, nine Lipizzaner, five Friesians, four Thoroughbreds, four ponies, four Arabians, and three American Breeds-aged 1 to 17 years (mean: $9.1 \pm 2.8$ years), with chronic respiratory symptoms such as cough, nasal discharge, dyspnea, or poor performance were included in this study. Chronicity of a minimum of 4 weeks was the minimum requirement for inclusion in the study. Most of the equine patients (76\%) were referred for a second opinion. The same standardized examination protocol was followed in all cases.

\subsection{Examination Protocol}

\subsubsection{History}

A special questionnaire was developed for taking the history. Breed, age, gender, usage of the horse, and a complete history with presenting signs, disease process, duration and type of previous treatments, and stabling conditions were recorded. Then, on the basis of these data, a simple scoring system was established to evaluate the stabling technology and disease process for statistical analysis (Table 1). The months of clinical admission for examination and disease establishment or exacerbations were noted. Referring surgeons were questioned about diagnostic techniques they used in each particular respiratory case and also about their suspected diagnosis.

\subsubsection{Clinical Examination}

A general physical examination was performed about 60 minutes after the horse arrived at the clinic. The main findings regarding the respiratory tract (RT) were evaluated with clinical scores on the basis of the methods developed by Naylor et al. [18] and Traub-Dargatz et al. with slight modifications [19] (Table 2). The sum of the numbers assigned to the different symptoms was used to generate the general clinical severity score.

\subsubsection{RT Endoscopy}

In the majority of the cases, RT endoscopy (CF-VL, Olympus GmbH, Hamburg, Germany) was performed without sedation to obtain most of the information about the function of both the lower and upper airways. In noncooperative animals, sedation with detomidine $(10 \mu \mathrm{g} /$ bwt; Domosedan inj.; Orion Pharma, Espoo, Finland) in combination with butorphanol (10 $\mu \mathrm{g} / \mathrm{bwt}$; Alvegesic inj.; Alvetra u. Werfft GmbH, Wien, Austria) was used. The nasal passages, pharynx, larynx, and guttural pouches were inspected and the upper respiratory tract (URT) was evaluated with score 0 if negative and with score 1 if any

Table 1

Simplified history questionnaire focusing on differentiation between environmental-induced and infectious disorders

\begin{tabular}{|c|c|c|c|c|c|}
\hline Score & $\begin{array}{l}\text { Duration of } \\
\text { Disease }\end{array}$ & Course of Disease & Stabling & Infection & $\begin{array}{l}\text { Treatment Steroid } \\
\text { Anti-Inflammatory Drug }\end{array}$ \\
\hline 0 & $>4$ weeks & Continuous signs & Pasture & $\begin{array}{l}\text { Fever, companion animals } \\
\text { were affected }\end{array}$ & No or negative reaction \\
\hline 1 & $>6$ weeks & & $\begin{array}{l}\text { Hypoallergenic bedding } \\
\text { and soaked hay }\end{array}$ & & No treatment \\
\hline 2 & $>8$ weeks & Remission-exacerbation & Simple stabling & $\begin{array}{l}\text { No fever, no other horse } \\
\text { affected }\end{array}$ & Positive reaction \\
\hline
\end{tabular}


Table 2

Clinical severity scoring system (according to Naylor et al. [18] and Traub-Dargatz et al. with modifications [19])

\begin{tabular}{llllll}
\hline Score & $\begin{array}{l}\text { Respiratory } \\
\text { Rate }\end{array}$ & Respiratory Effort & Lung Auscultation & Cough & Nasal Discharge \\
\hline 0 & $<20$ & No & Normal & No & No or serous \\
1 & $20-30$ & Increased & $\begin{array}{l}\text { Increased bronchial sounds } \\
\text { and abdominal lift }\end{array}$ & $\begin{array}{l}\text { Induced, strong } \\
\text { Spontaneous, frequent } \\
\text { or bouts }\end{array}$ & $\begin{array}{l}\text { Mucinous } \\
\text { Mucopurulent }\end{array}$ \\
3 & $30<$ & Flared nostrils and anal movement & $\begin{array}{c}\text { Generalized wheezes and crackles } \\
\text { or reduced lung sounds despite } \\
\text { deep breath }\end{array}$ & \\
& & & & \\
\hline
\end{tabular}

functional disorder was suspected. The volume of the respiratory secretion (RS) present in the cranial thoracic trachea was semiquantitatively described according to the grading system by Gerber et al. [20]. The nature of the RS was also recorded as mucoid, mucopurulent, purulent, or hemorrhagic. Tracheal and bronchial respiratory mucosa was also examined for evidence of inflammation, that is, for bluntness of the normally sharp carina and for the presence of hyperemia. End expiratory bronchoconstriction or bronchial collapse was also noted.

\subsubsection{Respiratory (Tracheal) Secretion Cytology and Culture}

RS was collected transendoscopically through the work channel using a sterile 2-m long plastic catheter (PW1V, Olympus GmbH, Hamburg, Germany). Within 1 hour of collection, an air-dried smear of RS was prepared and fixed with a fixative, and a differential cell count of 100 cells was performed on a Diff-Quick (Reagens Kft., Budapest, Hungary) stain preparation. The sample was sent for bacteriology when secretion was macroscopically considered purulent or the history had described a previous suspected RT infection or the results of clinical examinations were suspicious of infectious origin. Samples were injected to a transport media and sent for culturing to a specialized veterinary microbiology laboratory.

\subsubsection{Bronchoalveolar Lavage Fluid Cytology and Culture}

In each case, bronchoalveolar lavage fluid (BALF) was obtained through a BIVONA catheter (Bivona Medical Technologies Inc., Gary, IN) with horses under sedation as previously described. To reduce the physical irritation of the mucous membrane, $0.5 \%$ lidocaine solution (Lidokain inj.; Richter Gedeon Nyrt., Budapest, Hungary) was sprayed on the carina, and then $350 \mathrm{~mL}$ of lukewarm saline was instilled and aspirated. The volume of fluid gained back, its transparency, color, and the presence of a foamy layer were recorded. Within 30 minutes of collection, BALF cytospin cell preparations were made. Romanowsky stain (DiffQuik; Reagens Kft., Budapest, Hungary) was used, while keeping in mind that this stain has been found to be inadequate for detecting pulmonary mast cells [21,22]. Differential cell counts were performed on 300 cells by a board-certified clinical pathologist blinded to the clinical and endoscopic findings. Values given by Derksen et al. [23] were used as references.

In 67 cases of the supplementary laboratory examinations (48/67), further diagnostic imaging procedures (67/ 67 ) or bronchodilator test (10/67) with $0.02 \mathrm{mg} / \mathrm{kg}$ intravenous atropine (Atropinum sulfuricum inj., Egis, Budapest, Hungary) were performed (Table 3).

\subsection{Diagnostic Criteria Used to Classify Cases}

\subsubsection{RAO/Heaves}

RAO was defined as chronic neutrophilic pulmonary inflammation associated with the presence of hay and/or straw in the affected horses' environment and with clinical manifestations varying from mild cough to severe dyspnea at rest. The BALF of horses with RAO showed moderate to severe neutrophilia ( $>20 \%$ cells), decreased lymphocyte, and alveolar macrophage counts $[25,26]$. Summer pastureassociated (SPA)-RAO is clinically indistinguishable from RAO except that the affected horses develop signs while maintained on pasture [9].

\subsection{2. $I A D$}

By definition, horses with IAD might show poor performance, exercise intolerance, or coughing, with or

Table 3

Supplementary diagnostic procedures in selected cases (performed as described by Lekeux et al. [24])

\begin{tabular}{|c|c|c|}
\hline Type of Examination & $\begin{array}{l}\text { Number of } \\
\text { Tested Animals }\end{array}$ & Indication \\
\hline Thoracic radiography & 51 & Moderate or severe clinical signs \\
\hline Arterial blood gas analysis & 43 & Moderate or severe resting dyspnea or tachypnoea \\
\hline Thoracic ultrasonography & 35 & Distorted lung borders on percussion or positive thoracic radiography \\
\hline Hematology & 20 & History of fever, depression, or weight loss \\
\hline Serology & 12 & History of fever or more horses affected simultaneously nearby \\
\hline Culture on BALF & 12 & $\begin{array}{l}\text { History of fever, or suspected respiratory infections, or diffuse abnormal } \\
\text { lung patterns on thoracic radiographies }\end{array}$ \\
\hline $\begin{array}{l}\text { Bronchodilator (atropine) } \\
\text { administration }\end{array}$ & 10 & Severe dyspnea \\
\hline Treadmill endoscopy & 6 & Supposed dynamic URT disorders based on history or resting endoscopy findings \\
\hline Molecular diagnostic tests & 3 & Fever, nonresponsive to antibiotic treatments and interstitial radiographic pattern \\
\hline Lung biopsy & 1 & Nonresponsive to any treatment, nodular interstitial radiographic pattern \\
\hline
\end{tabular}

BALF, bronchoalveolar lavage fluid; URT, upper respiratory tract. 
without excess tracheal mucus, but without showing depression, fever, or increased respiratory efforts at rest [9]. It is commonly reported in young racehorses and decreases in frequency with increasing age [13], but nonracehorses of all ages can have IAD $[9,26]$. The most commonly encountered BALF cytologic profiles are characterized by increased total nucleated cell count with mild neutrophilia, lymphocytosis, monocytosis [7,9,26], or eosinophilia [27,28]. Although neutrophilic inflammation is commonly observed in BALF from horses both with RAO and IAD, the neutrophilia is usually less pronounced in cases of IAD (ie, $<20 \%$ ).

\subsubsection{Infectious Disorders}

Manifestations of infection such as lymphadenitis, fever, depression, decreased appetite, and weight loss are usually present in lower airway diseases of bacterial, viral, fungal, or parasitic origin [9]. Diagnosis is based on a positive culture with concurrent suggestive cytological findings (intracellular bacteria or fungal spores and signs of neutrophilic degeneration, like swollen nuclei or karyolysis) of tracheal wash fluid or an increase in antibody titer over the course of the disease within 14 to 21 days in suspected viral infection or a positive result of other molecular diagnostic tests.

\subsubsection{Upper Respiratory Tract Functional Disorders with Small Airway Inflammation}

Upper airway endoscopy at rest or during exercise allows for the identification of significant upper airway diseases. Concurrent abnormal bronchoalveolar lavage (BAL) cytology reflects lower airway inflammation. Horses with mild upper respiratory tract functional disorders (URTFD), expiratory dyspnea at rest, and BAL cytology of neutrophils of $>20 \%$ were classified as RAO cases and URTFD was evaluated as coincidence findings.

\subsubsection{Exercise-induced Pulmonary Hemorrhage}

EIPH occurs primarily in horses performing short periods of high intensity work. The diagnosis is based on finding blood after performing bronchoscopy [29] or by detecting increased hemosiderin content within alveolar macrophages [30,31].

\subsubsection{Chronic Interstitial Lung Diseases of Noninfectious Origin and Neoplasia}

The interstitial lung disease is generally unresponsive to antimicrobial and anti-inflammatory therapy. Thoracic radiographs commonly show severe, diffuse, or nodular interstitial pattern. A transthoracic lung biopsy is the definitive test for diagnosing chronic interstitial lung disease or neoplasia [32].

\subsubsection{Undifferentiated Pulmonary Disorders}

This group was composed of animals with detectable pulmonary disease where the diagnosis did not fall clearly into any of the aforementioned categories.

\subsection{Statistical Analysis}

To compare the history (Table 1 ) of horses with or without RAO, Fisher's test was used. To evaluate the usefulness or necessity of the different examination types used in the diagnostic workup of chronic equine lower airway and pulmonary cases, data were analyzed by using conditional inference tree methods [33]. First, we summed the historical and the clinical scores separately (scores are presented in Tables 1 and 2) and used these two new variables in a conditional inference tree model, which basically represents the decision-making paradigm frequently used in field veterinarian practice. Second, we added all the measured variables (age, gender, breed of the horse, historical data listed in Table 1, month of admission, clinical parameters listed in Table 2, RT endoscopy, RS cytology and bacteriology, BALF cytology, arterial blood gas and $\mathrm{pH}$ measurements, and $\mathrm{x}$-ray and ultrasound findings) into another conditional inference tree model to see how much the decision-making rule might improve by using these ancillary tests.

Conditional inference trees were constructed with

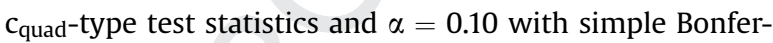
roni correction. Each split needed to send a minimum of $3 \%$ of the observations into each of the two child nodes. All analyses were performed using the $\mathrm{R}$ 2.7.2. Statistical Software [34].

\section{Results}

Overall, out of the 100 horses used in this study, 76 cases were referred by 45 veterinarians for a second opinion. They performed physical examination in all cases. RT endoscopy was carried out only in 22 cases with taking tracheal sample for culture and for cytology in 20 and eight cases, respectively. Blood was taken for hematology on 20 occasions, and BALF was sent for cytology on six occasions. Suspected diagnoses by field veterinarians were heaves (49/76) or respiratory infection (12/76), whereas the rest of patients were referred without any previous diagnosis.

On the basis of the BAL cytology, all of the examined 100 horses had some type and degree of lower airway disorder.

The case selection comprised horses with RAO $(\mathrm{n}=54)$, IAD ( $n=20)$, infectious pulmonary disease $(n=9)$, URTFD with SAI ( $n=13$; which consisted of idiopathic left laryngeal hemiplegia [ $n=4$ ], dorsal displacement of the soft palate $[n=4]$, pharyngeal collapse $[n=1]$, tracheal collapse $[\mathrm{n}=1]$, subglottic cyst $[\mathrm{n}=1]$, fourth branchial arch defect $\mathbf{Q} 2$ $[\mathrm{n}=1]$, and arytenoid chondritis [ $\mathrm{n}=1])$, and undifferentiated cases $(n=4)$. We did not group any animal as primary EIPH case, but we had horses with erythrophages in their BAL in all other groups except the undifferentiated one. During the examined period we did not diagnose any neoplasia or interstitial lung disease of noninfectious origin.

Chronic pulmonary disorders were more likely to be diagnosed during the warm months ( $87 \%$ of the cases were diagnosed between March and November), and most horses started to show symptoms or had exacerbated clinical signs also during this period. The distribution of the onset dates shows a trend for three main peaks during the year for patients with RAO and IAD: one peak at the beginning of spring, a second smaller peak in the middle of summer, and another peak at the end of summer (Fig. 1). Clinical admission dates clearly follow the peaks of onset.

The mean (SD) time span between the onset of the disease and the clinical admission was $4.4 \pm 3.7$ months, 


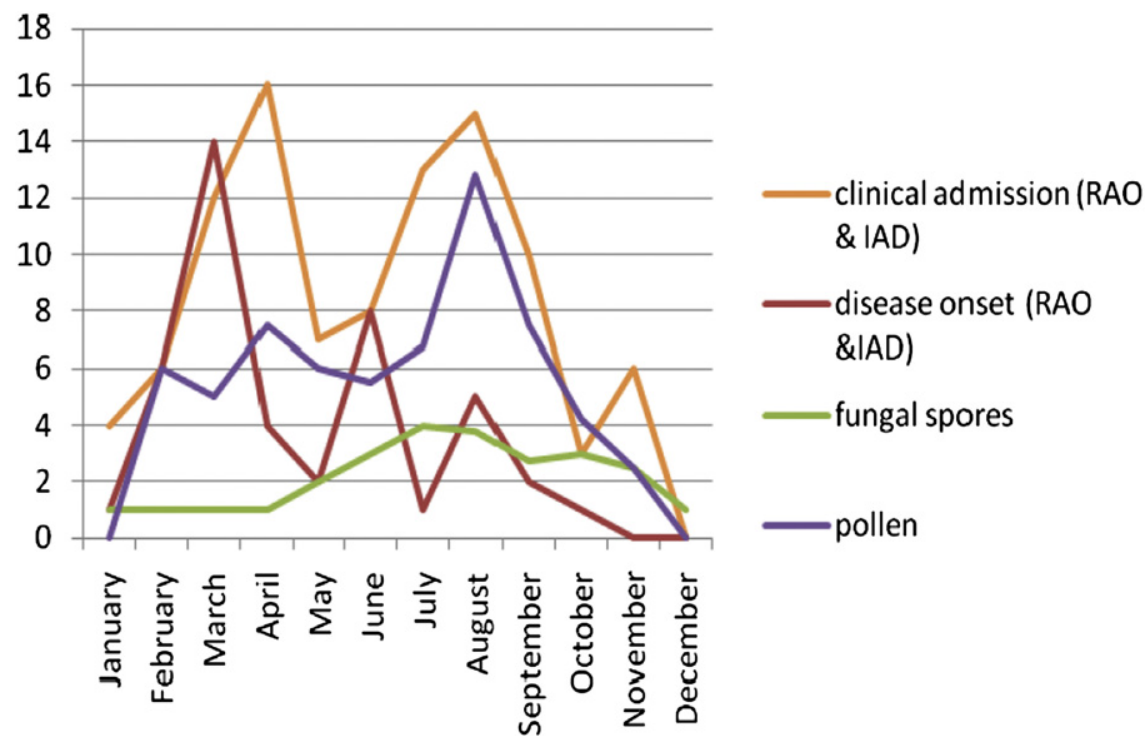

Fig. 1. The time-distribution of disease onset, clinical admission, and air-borne pollutants (fungal spores, pollen).

during which $66 \%$ of the animals were not treated at all or treated with no success (19\%).

Horses were used for pleasure riding (48\%) or sports (52\%), and the majority of them (71\%) were kept in stables with the traditional Hungarian stabling technology of feeding dry hay and bedding with straw. Some owners (24\%) had already implemented changes in this technology and introduced new hypoallergenic materials for bedding and used soaked hay. Only five horses, all of them diagnosed with RAO, were kept on pastures, but these animals also had supplementary dry hay almost year round.

Horses with RAO were significantly older as compared with those with $\operatorname{IAD}(P<.001)$, URTFD $(P=.022)$, or infectious disorders ([ID]; $P<.001)$. The average (SD) ages were 10.8 (2.7), 6.3 (1.4), 8.3 (3.8), and 6.0 (3.3) years, respectively. Horses with IAD, URTFD, or ID did not differ significantly from each other regarding age. Horses with RAO were 3.4 times more likely to have a duration of respiratory symptoms for $>8$ weeks (Fisher's test, $P=.022$ ), were 5.0 times more likely to show remission-exacerbation (Fisher's test, $P=.002$ ), and were 3.4 times more likely to show no fever (Fisher's test, $P=.023$ ), as compared with horses diagnosed with other chronic pulmonary disorders.

Regardless of the final diagnosis, the most common presenting clinical sign was cough (63\%), and the least common was poor performance (10\%). Nasal discharge and dyspnea were recorded in $41 \%$ and $40 \%$ of the cases, respectively.

The result of the first tree model (Fig. 2), in which we used the data usually available through history questionnaire and physical examination carried out by field practitioners in the classification of horses suffering from pulmonary disorders, suggests that horses with RAO will most likely be found among horses with summed clinical scores and summed historical scores $>4$. According to this tree model, 38 of the 54 RAO horses and five of nine ID horses could possibly be classified correctly as RAO or ID patient. However, 13 of the 46 non-RAO patients were also classified with this tree model as horses suffering from
RAO. None of the 20 IAD and 13 URTFD cases was classified correctly by this model.

For the second tree model, we added the data of ancillary diagnostic procedures (RT endoscopy: URT scoring and tracheal mucus grading, tracheal secretion cytology and bacteriology, BALF cytology, arterial blood gas measurements, as well as thoracic X-ray, and ultrasound). Results of URT endoscopy, neutrophil percentage in the BALF, history of previous infection, and age variables were selected as the main diagnostic criteria by the model (Fig. 3).

The first splitting criteria of the tree model resulted in a group of horses in which the endoscopy was positive for URTFD. A total of $76.5 \%$ of these horses had URTFD and $23.5 \%$ were diagnosed with RAO. All of the URTFD horses, except for $7 \%$ of the RAO horses, belonged to this group.

The second most important grouping variable was the neutrophil percentage in the BALF. In cases where endoscopy was negative and neutrophil granulocyte percentage was $<23 \%$, none of the horses were diagnosed with RAO.

Among horses that had negative URT endoscopy and a neutrophil granulocyte percentage $>23 \%$, RAO was most prevalent if horses had a history without fever and were aged $>6$ years ( $93 \%$ of the horses suffering from RAO were found in this group).

Within the group of horses with negative URT endoscopy, a neutrophil granulocyte percentage $>23 \%$ and a history of fever, $57 \%$ of horses were diagnosed with RAO, and $43 \%$ of them with ID (in total, $15 \%$ of horses diagnosed with RAO and 67\% with ID were found among this group of horses).

With the help of this tree model, 41 of the 54 RAO horses, 14 of 20 IAD horses, and all 13 URTFD horses were classified correctly as RAO, IAD, or URT patient. Only three of the 46 non-RAO patients were classified as horses suffering from RAO. None of the nine ID cases was classified correctly by this model.

Because of the small sample size, we were not able to present statistically significant correlations, but the results of further laboratory, diagnostic imaging, and other
878

879

880

881

882

883

884

885

886

887

888

889

890

891

892

893

894

895

896

897

898

899

900

901

902

903

904

905

906

907 


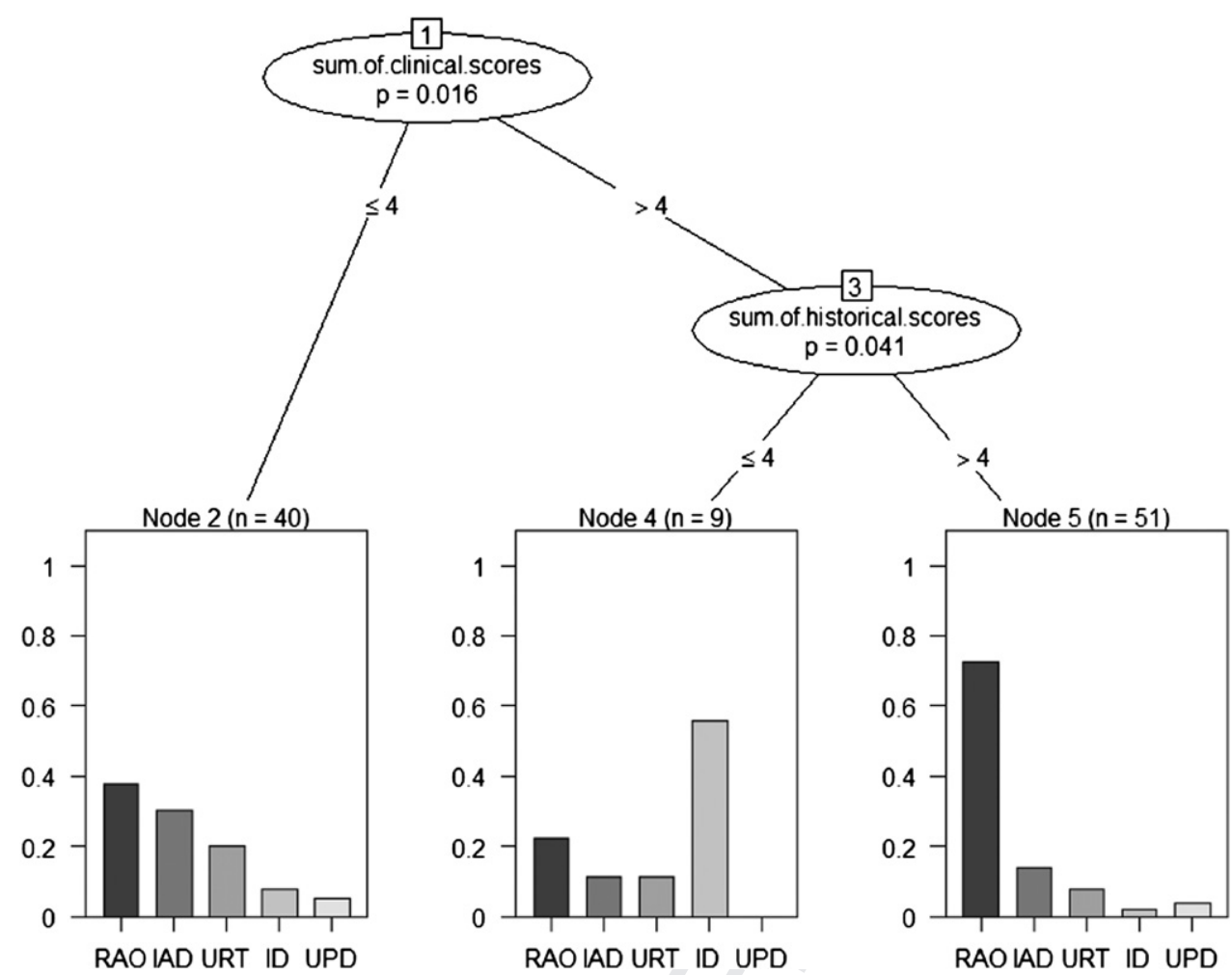

1044

1045

1046

1047

1048

1049

1050

1051

1052

1053

1054

1055

1056

1057

1058

1059

1060

1061

1062

1063

1064

1065

1066

1067

1068

1069

1070

1071

1072

1073

1074

1075

1076

1077

1078

1079

1080

1081

1082

1083

1084

1085

1086

1087

1088

1089

1090

1091

1092

1093

1094

1095

1096

1097

1098

1099

1100

1101

1102

1103

1104

1105 a significant problem in Hungarian climatic and geographic stables were open and most horses had some limited access to paddocks and pastures. 

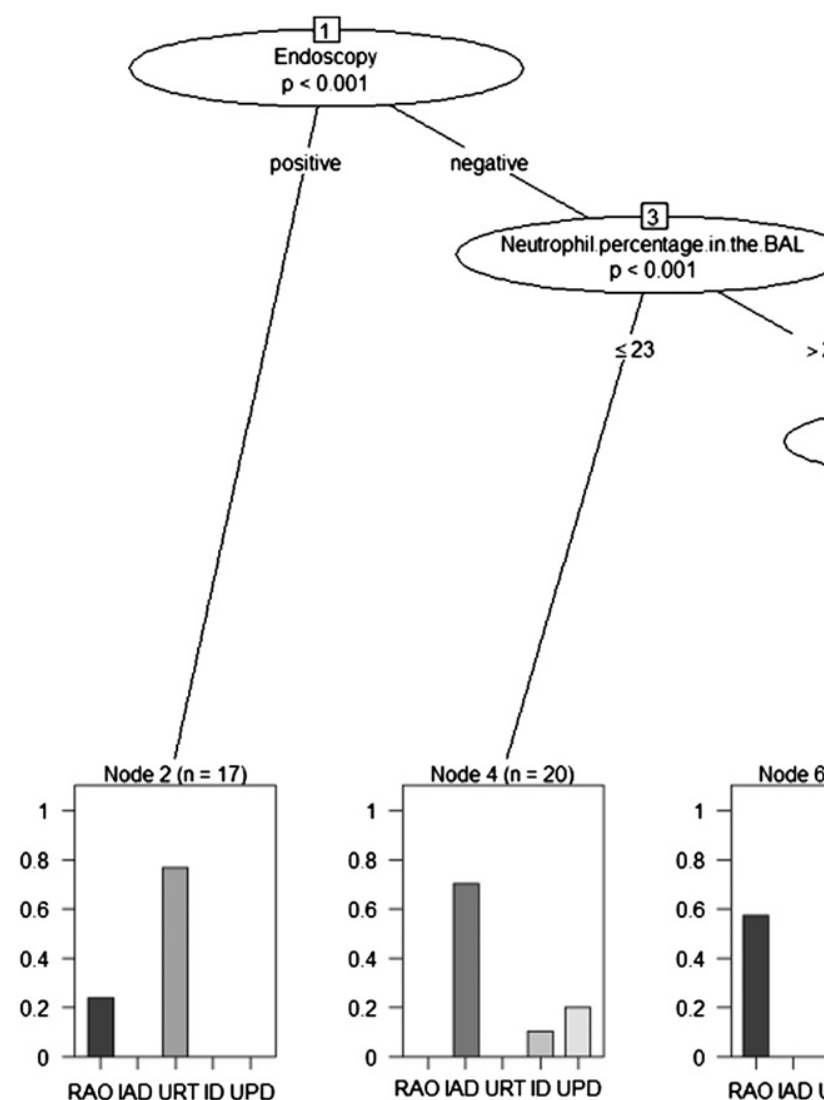

Fig. 3. Conditional inference tree built by using data when added the results of ancillary diagnostic procedures. RAO, recurrent airway obstruction; IAD, inflammatory airway disease; URTFD, upper respiratory tract functional disorders with small airway inflammation; ID, infectious disorders; UPD, undifferentiated Q7 pulmonary disorders.

conditions. Five horses in the RAO group were kept on pasture during the entire year and experienced exacerbation during the warm months, but the original onset of the disease occurred when these horses had previously been maintained in a stable environment. Although the field veterinarian directed these patients to pastures, however, environmental control was not successful in these cases because the climate of Hungary makes hay supplementation necessary even during the summer months. Both outdoor aeroallergens and dry hay supplementation might be responsible for these exacerbations.

As described in other studies, weeks to months may pass between the onset of clinical signs in the field and the time of clinical admission [2,38]. This might also influence our results regarding seasonal prevalence. Generally, more than 4 months passed during which the horses started representing clinical signs but were not examined, diagnosed, or treated at all. Subtle clinical signs usually do not alert owners and delay veterinary examination. This time delay might complicate diagnostic workup in several cases but makes RAO differentiation easier with recorded exacerbation-remission periods and relatively longer disease duration.

Details in the history about antecedent respiratory infection were not an exclusive feature of the cases in the ID group. We had horses in the IAD group that were referred as cases with suspected complications after some infection in the previous 6 months. Dixon et al [2] described that 19.2\% of COPD-affected horses had an infection immediately preceding the current respiratory disorder; in addition, according to Couëtil et al [11], owners often report a history of infectious respiratory disease in the months preceding the diagnosis of IAD with several horses in the stable being affected. The role of infectious agents in the development of RAO and IAD is still not clear $[6,9]$.

Although age is a helpful parameter when identifying cases of heaves, we also had horses in this group as young as 6 years of age. By contrast, our IAD group consisted of older horses than generally described in the previously published data $[9,40]$.

First opinion veterinarians usually have limited possibilities to perform special laboratory or any diagnostic imaging techniques. On the basis of the present study, a specific questionnaire regarding history and a thorough clinical examination can be reliable in diagnosing horses with RAO. Hotchkiss et al. [41] have previously demonstrated the usefulness of a well-constructed questionnaire in discriminating between horses with and without RAO. Another study also emphasized that the majority of cases with heaves can be correctly diagnosed on the basis of physical examination [18]. According to our study, these cases with typical history and unequivocal clinical signs of heaves accounted for $70 \%$ of the RAO patients, which accounted for only $38 \%$ of all cases. This reflects other 
Table 4

Additional findings of laboratory, diagnostic imaging, and other supplementary procedures

\begin{tabular}{|c|c|c|c|c|c|c|}
\hline \multirow[t]{2}{*}{ Type of Examination } & \multirow[t]{2}{*}{ Findings } & \multicolumn{5}{|c|}{ Number of positive cases/final diagnosis } \\
\hline & & RAO & IAD & ID & $\begin{array}{l}\text { URTFD } \\
\text { with SAID }\end{array}$ & Undifferentiated \\
\hline \multirow[t]{3}{*}{ Bronchoscopy } & Bronchoconstriction & 9 & & 1 & & 1 \\
\hline & Food particles in trachea & & & & 4 & \\
\hline & Hemorrhagic mucus in trachea & & & 2 & & \\
\hline Tracheal cytology & Septic inflammation & 2 & & 10 & 2 & \\
\hline Tracheal culture & $\begin{array}{l}\text { Streptococcus zooepid }(\mathrm{n}=4) \text {, Streptococcus } \\
\text { Equi }(\mathrm{n}=2), \text { Klebsiella pneumonia }(\mathrm{n}=1), \\
\text { Actinobacillus Equuli }(\mathrm{n}=1), \\
\text { Staphylococcus aureus }(\mathrm{n}=1)\end{array}$ & 2 & & 6 & 1 & \\
\hline \multirow[t]{2}{*}{ BALF cytology additional findings } & Hemosiderophages & 1 & 2 & 2 & 1 & \\
\hline & Intracellular plant or pollen particles & 4 & & 2 & 5 & \\
\hline BALF culture & Str zooepidemicus (1) & & & 1 & & \\
\hline \multirow[t]{5}{*}{ Thoracic radiology } & Severe bronchial pattern & 14 & & & & 1 \\
\hline & Increased radiolucency with concave diaphragm & 3 & & & & \\
\hline & Bronchiectasis & 2 & & & & \\
\hline & Increased interstitial-bronchial pattern & 6 & & 8 & 2 & 3 \\
\hline & Increased interstitial pattern & & 2 & 1 & & \\
\hline \multirow[t]{4}{*}{ Thoracic ultrasonography } & Caudally displaced lung borders & 5 & & & & \\
\hline & Cranially displaced lung borders & & & 1 & & 1 \\
\hline & Comet tail echoes & 5 & & 8 & 3 & 4 \\
\hline & Subpleural hypoechogenic areas & & & 4 & & \\
\hline \multirow[t]{2}{*}{ Bronchodilator administration } & Positive & 7 & & & & \\
\hline & Negative & 1 & & & & 2 \\
\hline Serology & EHV4 $(n=2)$, equine virusarteritis $(n=1)$ & & & 2 & 1 & \\
\hline \multirow[t]{3}{*}{ Hematology } & anemia & & & 1 & & \\
\hline & lymphocytosis & & & 1 & & \\
\hline & eosinophilia & & & & & 1 \\
\hline PCR & EHV5 & & & 1 & & \\
\hline Lung biopsy & Nodular fibrosis & & & 1 & & \\
\hline
\end{tabular}

RAO, recurrent airway obstruction; IAD, inflammatory airway disease; URTFD, upper respiratory tract functional disorders; SAID, small airway inflammatory Q10 disease.

previous data indicating that history taking and the results of physical examinations were not sufficient to establish a respiratory diagnosis [26,42]. None of the clinical signs were typical for any disorder. More severe respiratory symptoms were suggestive for RAO or ID, but history could help to differentiate between them.

Performing BAL and evaluating the cytology sample had a great effect on carrying out a successful diagnostic workup. Increased neutrophil counts are the main diagnostic criteria for RAO, but cutoff values vary greatly among publications depending on the BAL technique used or the population studied [31]. Because we had no previous data concerning our technique and horse population, we decided to possibly include equine patients in the RAO group with neutrophils of $>20 \%$. Finally, we realized that our cases with heaves had $>23 \%$ of neutrophils in their BALF. This finding is rather in agreement with the proposal that $>25 \%$ neutrophils in BAL are necessary for a horse to qualify as being affected with RAO [8]. Neutrophilia in the BAL sample was prominent in the RAO horses as well as in most of the ID-affected horses, which complicated differentiation on the basis of cytology. BAL cytology results combined with no previous febrile period history and the age of the horse resulted in the identification of most of the patients with RAO.

Although lower airway disorders cannot be differentiated on the basis of RT endoscopy, endoscopy is the unique method to define URTFD [43,44]. Simple respiratory endoscopy at rest selected all URTFD cases. In our caseload, none of the animals presented with suspicion of upper airway dysfunction. Cough, nasal discharge, and dyspnea can be caused by upper airway inflammation and obstruction as well $[9,45]$. Interestingly, all of the horses with URTFD also had abnormal BAL cytology results indicating small airway involvement, which might also be responsible for the clinical signs. Small airway inflammation (SAI) in these cases could have possibly been caused by the altered airflow dynamics causing decreased mucociliary clearance and more negative pressure in the lower airway segments resulting in mechanical irritation or hemorrhage. Mild chronic aspiration and secondary infections could contribute to the disease. In a previous study about URT functional problems, the authors also suggested their predisposing role in lower airway inflammation [46]. Depending on each individual case, we have found various cytological findings with different increased cellular ratios. Increased neutrophil and exfoliated epithelial cell ratios can be explained by mechanical irritation or septic inflammation. Neutrophilia could be a sign of concurrent RAO as well. Aspiration of foreign material and bleeding can cause an increased number of macrophages [47]. Horses with dorsal displacement of the soft palate had either high lymphocyte or high neutrophil numbers in BAL fluid, both of which might be a sign of chronic viral or bacterial infection. Chronic bacterial or viral infections can cause upper airway inflammation simultaneously and may result in impaired function of the soft palate $[14,48]$. In these cases, SAI might result from URT dysfunction or simply be a concurrent finding. Holcombe et al. [49] demonstrated that upper and lower airway 
inflammations were both associated with stabling, but there was no direct correlation between them.

We performed further ancillary diagnostic tests in 67 animals but according to the inference tree method, these tests were not necessary for grouping them reliably in $67 \%$ of cases. These methods were useful only to refine the final diagnosis. In all other cases (22/100), a very thorough and complex diagnostic workup using special laboratory tests and diagnostic imaging techniques was necessary to reach the final diagnosis, thereby making it unachievable for the field veterinarian.

Radiographic and ultrasonographic evaluation of the chest facilitated differentiating mainly infectious conditions; all of them in this group had abnormal lung pattern and ultrasonographic findings. As described previously, these imaging techniques are helpful in differentiating between horses in group IAD and ID [9]; however, in the absence of clinical evidence of more extensive, infectious disease, thoracic radiographs neither refine nor improve the diagnosis of IAD, but only increase diagnostic costs [50]. None of the radiologic findings were pathognomonic, not even in the ID group; thus, in each case, further diagnostic procedures were necessary. Caudally displaced lung borders, increased radiolucency with concave diaphragm, and bronchiectasis were sequelae of severe RAO, as demonstrated earlier [6,51].

Septic tracheal cytology or positive culture did not mean that animals could simply be grouped in the infectious group. Two horses with positive culture were placed in the URTFD group based on endoscopic results, and two horses with a secondary infection were placed in the RAO group based on history, clinical signs, and positive bronchodilator test. When infections complicated the suspected RAO cases, bronchoconstriction, as one of the causes of the respiratory signs and its severity, was evaluated with the atropin test [52]. Bacteria are commonly detected in airways of horses affected with heaves, and in many cases these findings are caused by impaired clearance as a result of RAO [2,53,54]. Further, infectious cases could be identified on the basis of Q4 serology and PCR, but hematology did not prove to be reliable. Blood gas parameters did not differ significantly between groups, being quite useful for evaluating the evolution stage of the inflammatory process rather than in the diagnostic workup [55].

Finally, $4 \%$ of the equine patients were left without a specific diagnosis. In such cases, conclusions were drawn from the response to different treatment protocols. In two cases, we had contradictive results with history and clinical signs being typical for RAO but BAL cytology showing a low number of neutrophils. These horses improved with steroid treatment. These cases could be horses suffering from RAO in remission but because they did not fulfill the criteria of RAO definition, we had to handle them separately. This also points out the fact that horses with RAO in remission are difficult to evaluate and final diagnosis can only be based on the characteristic history and clinical signs. There were cases resembling ID, but all cytology, cultures, serology, and PCR examinations were negative. BALF cytology showed moderately increased neutrophil number and bronchoconstriction tests were negative. They responded well for rest, anti-inflammatory, and long-term antimicrobial treatment. In the undifferentiated cases mentioned earlier in the text, results of further ancillary diagnostic tests were not specific for any lower respiratory disorder. Inappropriate staining technique might also be responsible for some of the unidentified cases.

We did not have any primary EIPH case, probably because we had not got any racing Thoroughbreds for examination and also other sports where EIPH is rather common, such as cutting, reining, polo, or cross-country event, are not widespread in Hungary. Hemosiderophages were found in some horses with all other types of disorders secondary to inflammation or obstruction of the airways. Frequent concurrent finding of hemosiderophages in BALF and tracheal mucopurulent secretion had earlier been demonstrated and supports the hypothesis of correlation between EIPH and lower airway inflammation [56,57].

The single chronic interstitial lung disease diagnosed was the equine multinodular pulmonary fibrosis, but because equine herpes virus 5 was detected with PCR, in this case we classified it as ID. Other chronic interstitial lung diseases were not identified, thus, they seem to account for minimal percentage of respiratory cases.

\section{Conclusions}

We recorded that first opinion veterinary surgeons infrequently use ancillary diagnostic techniques when investigating chronic lower airway disorders. We conclude that taking the history and performing a clinical examination are not sufficient to establish a final diagnosis in these cases. Number of successful diagnostic workup in the field would be higher if first opinion veterinarians used RT endoscopy and broncholaveolar lavage as basic diagnostic tools in all chronic respiratory cases. These ancillary diagnostic procedures are easily performed in stable circumstances as well. Although tracheal secretions and blood samples are more easily collected, results of TS cytology Q 5 and hematology are less informative. Culturing tracheal lavage samples might add to the final diagnosis but results cannot be evaluated easily because secondary infections can complicate primary non-ID and false negative cultures may also occur.

We also concluded that establishing a diagnosis in stable circumstances is impossible in approximately onequarter of cases, and it is still challenging in clinical settings.

It is also noteworthy that RAO appears to be widespread in Hungary, accounting for more than half of chronic pulmonary disorders. Finally, contrary to the current published data, it interestingly occurs mainly during the warm season. The high outdoor dust, air-borne mold, and pollen levels, and the necessary hay supplementation on pastures during the warm months complicate the optimal management of horses with RAO and commonly induce exacerbations in this period of the year.

\section{Acknowledgments}

The authors thank the referring veterinarians and all of their colleagues from the Clinic for Large Animals for their contributions and assistance. Special thanks are given to Timea Dufek for helping with the paperwork and keeping 
the files updated and Veronika Kokai for preparing for all the diagnostic procedures.

\section{References}

[1] Dixon PM, McGorum BC, Pirie RS. Inflammatory airway disease: European clinicians' perspective. In: Hoffman A, Robinson NE, Wade JF. Proceedings of a workshop on inflammatory airway disease: defining the syndrome. Newmarket: R \& W Publications; 2003, p. 78. Havemeyer Foundation Monograph Series No. 9.

[2] Dixon PM, Railton DI, McGorum BC. Equine pulmonary disease: a case controlled study of 300 referred cases. Part 1: examination techniques, diagnostic criteria and diagnoses. Equine Vet J 1995;27:416-21.

[3] Ward MP, Couëtil LL. Climatic and aeroallergen risk factors for chronic obstructive pulmonary disease in horses. Am J Vet Res 2005;66:818-24.

[4] Horohov DW, Mills WR, Gluck M. Specific and innate immunity in the lung as it relates to equine RAO. In: Tessier C, Gerber V, editors. Fourth world equine airways symposium (WEAS). Berne, Switzerland: Pabst Science Publishers; 2009. p. 106-8.

[5] Gerber V, Swinburne JE, Blott SC, Nussbaumer P, Ramseyer A, Klukowska-Rotzler J, et al. Genetics of recurrent airway obstruction (RAO). Dtsch Tierarztl Wochenschr 2008;115:271-5.

[6] Lavoie JP. Recurrent airway obstruction (Heaves) and summerpasture-associated obstructive pulmonary disease. In: Dixon PM, McGorum BC, Schumacher J, Robinson NE, editors. Equine Respiratory medicine and surgery. London: Saunders Co Ltd; 2007. p. 565-91.

[7] Moore RB, Krakowka S, Robertson JT, Cummins JM. Cytologic evaluation of bronchoalveolar lavage fluid obtained from Standardbred racehorses with inflammatory airway disease. Am J Vet Res 1995;56:562-7.

[8] Robinson N. International workshop on equine chronic airway disease. Equine Vet J 2001;33:5-19.

[9] Couëtil LL, Hoffman AM, Hodgson J, Buechner-Maxwell V, Viel L, Wood JLN, et al. ACVIM consensus statement: inflammatory airway disease of horses. J Vet Intern Med 2007;21:356-61.

[10] Viel L. Small airway disease as a vanguard for chronic obstructive pulmonary disease. Vet Clin North Am Equine Pract 1997;13:549-60.

[11] Couëtil LL. IAD: cough, poor performance, mucus in the airways-what is so important about that? AAEP Proc 2002;48:200-7.

[12] Holcombe S, Jackson C, Gerber V, Jefcoat A, Berney C, Eberhardt S, et al. Stabling is associated with airway inflammation in young Arabian horses. Equine Vet J 2001;33:244-9.

[13] Wood JLN, Newton JR, Chanter N, Mumford J. The association between respiratory disease and bacterial and viral infections in British racehorses. J Clin Microbiol 2005:43:120-6.

[14] Rush B, Mair T. Equine respiratory diseases. 1st ed. Oxford: Blackwell Science Ltd; 2004

[15] Couëtil LL, Hinchcliff KW. Non-infectious diseases of the lower respiratory tract. In: Hinchcliff KW, Kaneps AJ, Geor RJ, editors. Equine sports medicine and surgery. London: Saunders; 2004. p. 613-57.

[16] Cook WR, Williams RM, Kirker-Head CA. Upper airway obstruction (partial asphyxia) as the possible cause of exercise-induced pulmonary hemorrhage in the horse: an hypothesis. J Equine Vet Sci 1988;8:11-26.

[17] Langsetmo I, Fedde MR, Meyer TS, Erickson HH. Relationship of pulmonary arterial pressure to pulmonary haemorrhage in exercising horses. Equine Vet J 2000;32:379-84.

[18] Naylor JM, Clark EG, Clayton HM. Chronic obstructive pulmonary disease: usefulness of clinical signs, bronchoalveolar lavage, and lung biopsy as diagnostic and prognostic aids. Can Vet J 1992;33: 591-8.

[19] Traub-Dargatz JL, McKinnon AO, Thrall MA, Jones RL, Bruyninckx W, Blancquaert AM, et al. Evaluation of clinical signs of disease, bronchoalveolar and tracheal wash analysis, and arterial blood gas tensions in 13 horses with chronic obstructive pulmonary disease treated with prednisone, methyl sulfonmethane, and clenbuterol hydrochloride. Am J Vet Res 1992;53:1908-16.

[20] Gerber V, Straub R, Marti E, Hauptman J, Herholz C, King M, et al. Endoscopic scoring of mucus quantity and quality: observer and horse variance and relationship to inflammation, mucus viscoelasticity and volume. Equine Vet J 2004;36:576-82.

[21] Hughes KJ, Malikides N, Hodgson DR, Hodgson JL. Comparison of tracheal aspirates and bronchoalveolar lavage in racehorses 1 . Evaluation of cytological stains and the percentage of mast cells and eosinophils. Aust Vet J 2003;81:681-4.
[22] Leclere M, Desnoyers M, Beauchamp G, Lavoie JP. Comparison of four staining methods for detection of mast cells in equine bronchoalveolar lavage fluid. J Vet Intern Med 2006;20:377-81.

[23] Derksen FJ, Brown CM, Sonea I, Darien BJ, Robinson NE. Comparison of transtracheal aspirate and bronchoalveolar lavage cytology in 50 horses with chronic lung disease. Equine Vet J 1989;21:23-6.

[24] Lekeux P. Equine respiratory diseases. Ithaca, NY: International Veterinary Information Service (www.ivis.org); 2002.

[25] Derksen FJ, Scott JS, Miller DC, Slocombe RF, Robinson NE. Bronchoalveolar lavage in ponies with recurrent airway obstruction (heaves). Am Rev Respir Dis 1985;132:1066-70.

[26] Couëtil LL, Rosenthal FS, DeNicola DB, Chilcoat CD. Clinical signs, evaluation of bronchoalveolar lavage fluid, and assessment of pulmonary function in horses with inflammatory respiratory disease. Am J Vet Res 2001;62:538-46.

[27] Hare JE, Viel L. Pulmonary eosinophilia associated with increased airway responsiveness in young racing horses. J Vet Intern Med 1998; $12: 163-70$

[28] Hoffman AM. Bronchoalveolar lavage technique and cytological diagnosis of small airway inflammatory disease. Equine Vet Edu 1999;11:208-14.

[29] Raphel CF, Soma LR. Exercise-induced pulmonary hemorrhage in Thoroughbreds after racing and breezing. Am J Vet Res 1982;43:1123-7.

[30] Fogarty U. A bronchoalveolar lavage technique for routine diagnostic purposes. Equine Vet Edu 1990;2:102-4.

[31] Richard EA, Fortier GD, Lekeux PM, Van Erck E. Laboratory findings in respiratory fluids of the poorly-performing horse. Vet J 2010;185:115-22.

[32] Nolen-Walston RD, Sweeney CR. Equine restrictive lung disease, part 3: interstitial diseases. In: Lekeux P, editor. Equine respiratory diseases. Ithaca, NY: International Veterinary Information Service (www.ivis.org); 2002. B0308.0602.

[33] Nagy K, Reiczigel J, Harnos A, Schrott A, Kabai P. Tree-based methods as an alternative to logistic regression in revealing risk factors of crib-biting in horses. J Equine Vet Sci 2010;31:21-6.

[34] R Development Core Team. R, A language and environment for statistical computing. Available at: http://www.r-project.org. Vienna, Austria: R Foundation for Statistical Computing; 2007.

[35] Robinson NE, Derksen FJ, Olszewski MA, Buechner-Maxwell VA. The pathogenesis of chronic obstructive pulmonary disease of horses. $\mathrm{Br}$ Vet J 1996;152:283-306.

[36] Bracher V, von Fellenberg R, Winder CN, Gruenig G, Hermann M, Kraehenmann A. An investigation of the incidence of chronic obstructive pulmonary disease (COPD) in random populations of Swiss horses. Equine Vet J 1991;23:136-41.

[37] Davis E, Rush BR. Equine recurrent airway obstruction: pathogenesis, diagnosis, and patient management. Vet Clin North Am Equine Pract 2002;18:453-67.

[38] Couëtil LL, Ward MP. Analysis of risk factors for recurrent airway obstruction in North American horses: 1,444 cases (1990-1999). J Am Vet Med Assoc 2003;223:1645-50.

[39] Available at: http://www.nepegeszseg.net/pollenjelentes.php-2007.

[40] Chapman PS, Green C, Main JPM, Taylor PM, Cunningham FM, Cook AJ, et al. Retrospective study of the relationships between age, inflammation and the isolation of bacteria from the lower respiratory tract of thoroughbred horses. Vet Rec 2000;146:91-5.

[41] Hotchkiss JW, Reid SW, Christley R. Construction and validation of a risk-screening questionnaire for the investigation of recurrent airway obstruction in epidemiological studies of horse populations in Great Britain. Prev Vet Med 2006;75:8-21.

[42] Hoffman AM, Mazan MR. Airway obstruction and hyper-reactivity in horses with signs of inflammatory airway disease. In: Hoffman AM, Robinson NE, Wade JF, editors. Inflammatory airway disease: defining the syndrome. Newmarket: R\&W Publications; 2003. p. 71-4.

[43] Franklin SH, Naylor JR, Lane JG. Videoendoscopic evaluation of the upper respiratory tract in 93 sport horses during exercise testing on a high-speed treadmill. Equine Vet J Suppl 2006;36:540-5.

[44] Davidson EJ, Martin BB. Jr. Diagnosis of upper respiratory tract diseases in the performance horse. Vet Clin North Am Equine Pract 2003;19:51-62.

[45] Holcombe SJ, Ducharme GD. Abnormalities of the upper airway. In: Hinchcliff KW, Kaneps AJ, Geor RJ, editors. Equine sports medicine and surgery. London: Saunders; 2004. p. 613-57.

[46] Brakenhoff JE, Holcombe SJ, Hauptman JG, Smith HK, Nickels FA, Caron JP. The prevalence of laryngeal disease in a large population of competition draft horses. Vet Surg 2006;35:579-83.

[47] Hewson J, Viel L. Sampling, microbiology and cytology of the respiratory tract. In: Lekeux P, editor. Equine respiratory diseases. Ithaca, NY: International Veterinary Information Service (www.ivis. org); 2002. B0308.0602. 
[48] Holcombe SJ, Derksen FJ, Stick JA, Robinson NE. Pathophysiology of dorsal displacement of the soft palate in horses. Equine Vet J Suppl 1999;30:45-8.

[49] Holcombe SJ, Robinson NE, Jackson C, Berney C, Gerber V, Jefcoat A. Stabling, airway inflammation, and dorsal displacement of the soft palate in young horses. AAEP Proc 2000;46:254-5.

[50] Mazan MR, Vin R, Hoffman AM. Radiographic scoring lacks predictive value in inflammatory airway disease. Equine Vet J 2005;37:541-5.

[51] Lavoie JP, Dalle S, Breton L, Pierre H. Bronchiectasis in three adult horses with heaves. J Vet Int Med 2004;18:757-60.

[52] Robinson NE. Recurent Airway Obstruction (heaves). In: Lekeux P, editor. Equine respiratory diseases. Ithaca, NY: International Veterinary Information Service (www.ivis.org); 2002. B0308.0602.

[53] Couetil LL, Rosenthal FS, de Nicola DB, Chilcoat CD. Clinical signs, evaluation of bronchoalveolar lavage fluid, and assessment of pulmonary function in horses with inflammatory respiratory disease. Am J Vet Res 2001;62:538-46.

[54] McPherson EA, Thomson JR. Chronic obstructive pulmonary disease in the horse. 1: nature of the disease. Equine Vet J 1983;15: 203-6.

[55] Ferro E, Ferrucci F, Zucca E, DiFabio V, Castoldi S. Arterial blood gas analysis in 53 racehorses with a diagnosis of small airway inflammatory disease (SAID). J Equine Vet Sci 2002;22:165-8.

[56] Ferrucci F, Stancari G, Zucca E, Ayalon S, Falcone C, Ferro E. Specificity and sensitivity of ultrasonography and endoscopy for the diagnosis of exercise-induced pulmonary haemorrhage (EIPH) in 157 race horses. Vet Res Commun 2009;33(Suppl 1):185-8.

[57] Derksen FJ, Williams KJ, Uhal BD, Slocombe RF, De Feijter-Rupp H, Eberhart S, et al. Pulmonary response to airway instillation of autologous blood in horses. Equine Vet J 2007;39:334-9.
1995

1996

1997

1998

1999

2000

2001

2002

2003

2004

2005

2006

2007

2008

2009

2010

2011

2012

2013

2014

2015

2016

2017

2018

2019

2020

2021

2022

2023

2024

2025

2026

2027

2028

2029

2030

2031

2032

2033

2034

2035

2036

2037

2038

2039

2040

2041

2042

2043

2044

2045

2046

2047

2048

2049

2050

2051

2052

2053

2054

2055

2056 


\section{AUTHOR QUERY FORM}

\begin{tabular}{|c|c|c|}
\hline ELSEVIER & Article Number: 1159 & $\begin{array}{l}\text { Please e-mail or fax your responses and any corrections to: } \\
\text { E-mail: elsjmYJEVS@cadmus.com } \\
\text { Fax: 717-738-9479 or 717-738-9478 }\end{array}$ \\
\hline
\end{tabular}

Dear Author,

Please check your proof carefully and mark all corrections at the appropriate place in the proof (e.g., by using on-screen annotation in the PDF file) or compile them in a separate list. To ensure fast publication of your paper please return your corrections within 48 hours.

For correction or revision of any artwork, please consult http://www.elsevier.com/artworkinstructions.

Any queries or remarks that have arisen during the processing of your manuscript are listed below and highlighted by flags in the proof.

\begin{tabular}{|l|l|}
\hline $\begin{array}{c}\text { Location } \\
\text { in article }\end{array}$ & \multicolumn{1}{|c|}{$\begin{array}{c}\text { Query / Remark: Click on the Q link to find the query's location in text } \\
\text { Please insert your reply or correction at the corresponding line in the proof }\end{array}$} \\
\hline Q1 & Please provide with the manufacturer's details for "R 2.7.2. Statistical Software. \\
Q2 & $\begin{array}{l}\text { Please check whether the change made from "subepiglottic" to "subglottic" is correct. } \\
\text { Q3 }\end{array}$ \\
Q1ease spell out "COPD" in the text. \\
Q5 & Please spell out "PCR" in the text. \\
Q6 & Please spell out "TS" in the text. \\
Q7 & Please note that references have been renumbered to ensure sequential order in the text. \\
Q8 & ancillary diagnostic procedures were added. \\
Q9 & Please check whether all the genus and species as mentioned in the table are correct. \\
Q10 & Please check whether "SAID" has been correctly spelled out in the text. \\
\hline
\end{tabular}

Thank you for your assistance. 